\title{
Two clinical cases of granulomatosis with polyangiitis with isolated otitis media and mastoiditis
}

\author{
Georgi Kukushev¹, Desislava Kalinova², Ivan Sheytanov², Rasho Rashkov² \\ ${ }^{1}$ Department of Otolaryngology, Military Medical Academy, Sofia, Bulgaria \\ ${ }^{2}$ MHAT "St. Ivan Rilski", Clinic of Rheumatology, Medical University, Sofia, Bulgaria
}

\begin{abstract}
Granulomatosis with polyangiitis (GPA) is characterised by granulomatous necrotising inflammatory lesions of the upper and lower respiratory tract, often associated with pauci-immune glomerulonephritis. The diagnosis of granulomatosis with polyangiitis is made according to the classification criteria of the ACR criteria for granulomatosis with polyangiitis. We present two cases of granulomatosis with polyangiitis limited/localised form. The common feature between two clinical cases were not sufficient criteria for a definite diagnosis at the beginning. In both cases the clinical presence was otitis media with acute mastoiditis, peripheral facial nerve palsy, and severe headache. Early diagnosis and treatment of patients with granulomatosis with polyangiitis define favourable prognosis. On the other hand, the treatment of granulomatosis with polyangiitis (corticosteroids and immunosuppressive therapy) has various side effects, and the "ex juvantibus" therapy is hazardous.
\end{abstract}

Key words: otitis media, mastoiditis, granulomatosis with polyangiitis.

\section{Introduction}

Granulomatosis with polyangiitis (GPA) is characterised by granulomatous necrotising inflammatory lesions of the upper and/or lower respiratory tract, often associated with pauci-immune glomerulonephritis [1]. Granulomatosis with polyangiitis is also included in ANCA-associated vasculitis (AVV) in which anti-neutrophil cytoplasmic antibodies occur, and in the case of GPA they are antibodies to proteinase 3 [PR3] (anti c-ANCA antibodies).

Granulomatous ear, nose, and throat (ENT) lesions are the most typical manifestations of the disease, noted in more than three-quarters of patients at diagnosis, with crusting rhinitis, sinusitis, chronic otitis media, saddle-nose deformity, and/or nasal septum perforation $[2,3]$. Two different forms of granulomatosis with polyangiitis may be distinguished: systemic/generalised forms and localised/limited forms. Limited disease corresponds to GPA whose manifestations remain limited mostly to the upper respiratory tract [1]. Notably, these forms seem also to differ histologically, with systemic
GPA being associated more often with vasculitis lesions and localised GPA with marked granulomatous features [4]. The limited/localised forms represent less than onethird of all GPA cases at diagnosis and occur especially in women, and at a slightly younger age than in those with systemic GPA [4]. Its isolated otolaryngology manifestation is uncommon for this disease [2, 3].

The diagnosis is made according to the criteria of the ACR criteria for granulomatosis with polyangiitis [5-7].

We present two clinical cases of granulomatosis with polyangiitis with similar clinical manifestations but completely different laboratory and pathological presentations. In both cases the clinical presence was otitis media with acute mastoiditis, peripheral facial nerve palsy, and severe headache. The common feature between two clinical cases were insufficient criteria for a definite diagnosis at the beginning.

\section{Clinical case 1}

The patient, a 32-year-old man, presented with decreased hearing in the left ear, followed by ear drainage.

Address for correspondence:

Desislava Kalinova, MHAT “St. Ivan Rilski”, Clinic of Rheumatology, Medical University, Urvich street 13, 161 Sofia, Bulgaria,

e-mail: desi.kalinova@abv.bg

Submitted: 3.09.2017; Accepted: 16.10.2017 
Twelve days later the patient presented to the otolaryngologist again with ipsilateral facial nerve palsy and strong headache. Antibiotic therapy was started - ceftriaxone $2 \mathrm{~g}$ /day i.v. and metronidazole $2 \times 500 \mathrm{mg}$ i.v. The methylprednisolone was started in a dose of $40 \mathrm{mg}$ daily. A computed tomography of the head was performed revealing left mastoiditis (Fig. 1).

After the end of the antibiotic course and applied corticosteroids, because of the progressive clinical manifestations of the acute mastoiditis, a radical "wall down" mastoidectomy was performed.

The surgical procedure had three main purposes:

1. Eradication of the process because of the inner ear and facial nerve involvement.

2. Biopsy of the tissue in mastoid.

3. Control of the process (by performing "wall down" mastoidectomy the ENT surgeon has visual access to the open mastoid cavity through the drilled posterior wall of the external ear canal).
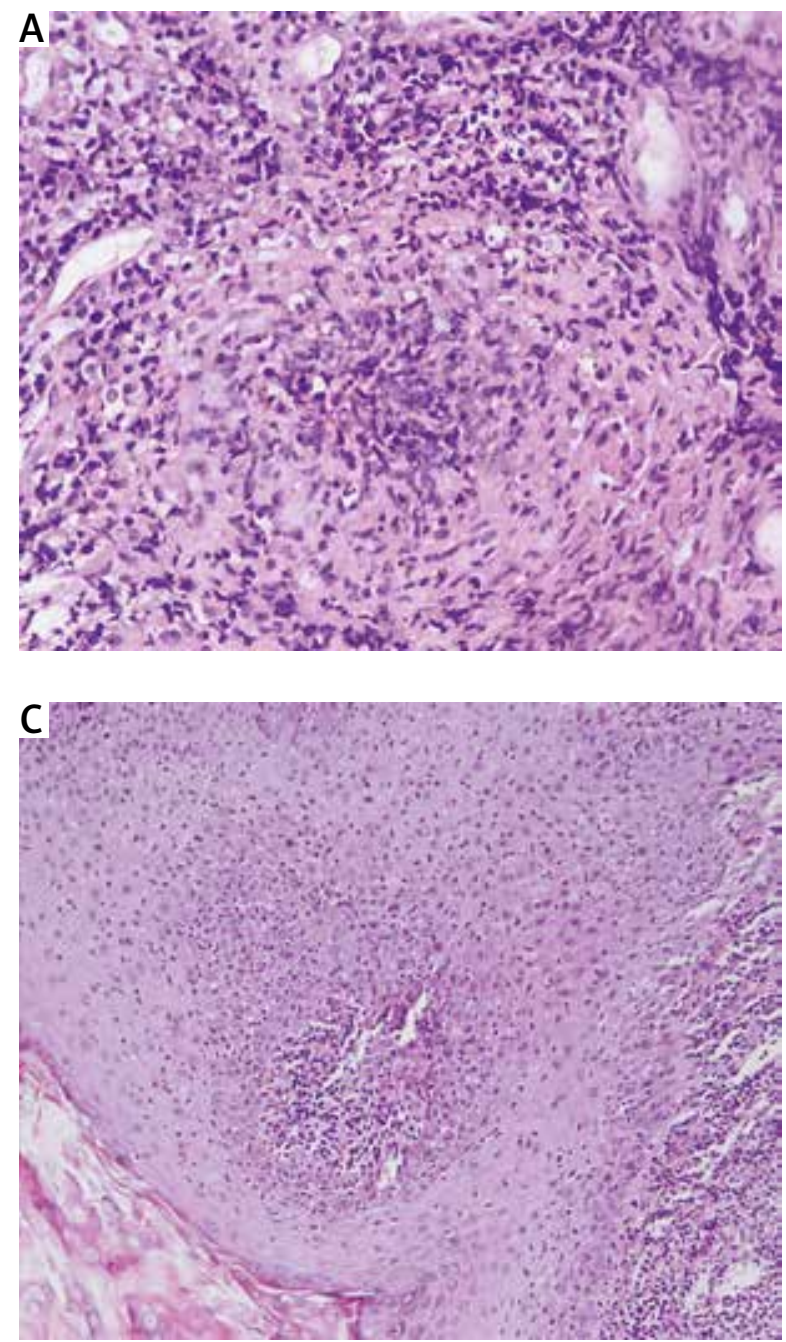

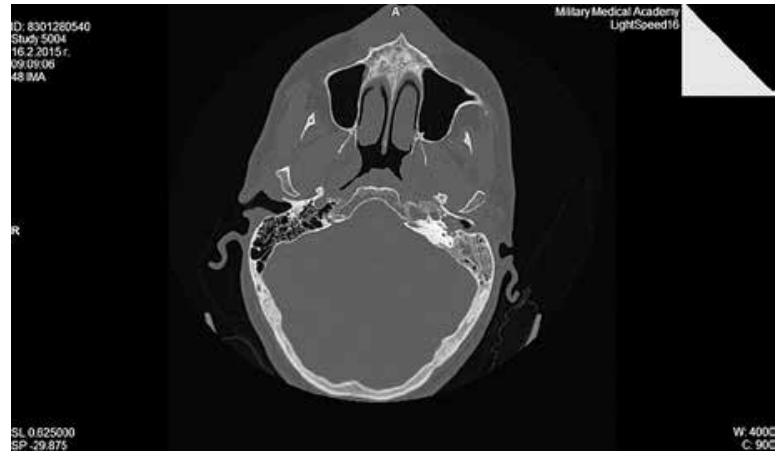

Fig. 1. Computed tomography of the head. Left mastoiditis and inflammation in the left middle ear cavity.

The middle ear as well as the mastoid was obliterated by granulomatous tissue because the biopsy showed a marked granulomatous necrotising inflammation (Fig. $2 \mathrm{~A}-\mathrm{C})$.

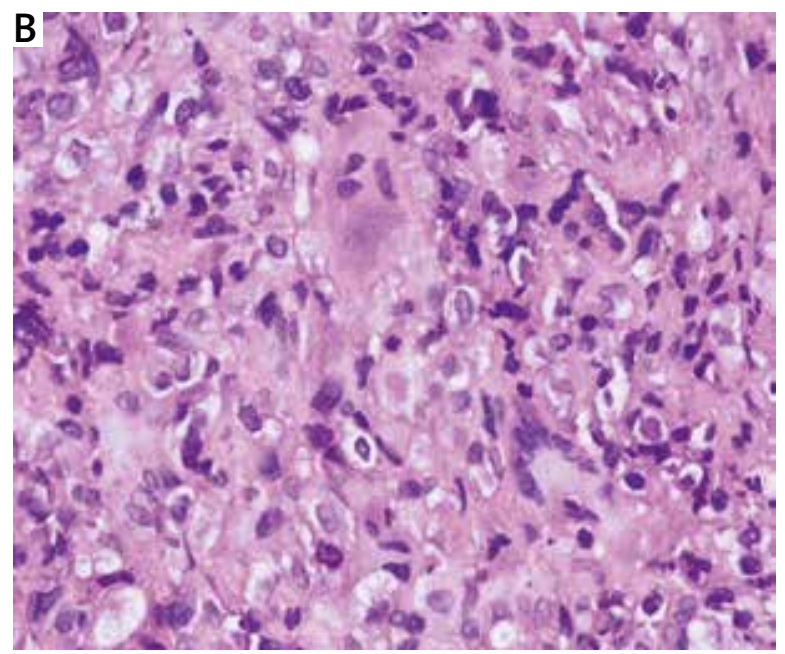

Fig. 2. Fragments of soft tissues, some covered with stratified (flattened) squamous epithelium with extensive zones of oedema, haemorrhage, and necrosis, and marked granulomatous necrotising inflammation. In the central area of the granuloma, necrosis and a cluster of lymphocytes were found without multinucleated giant cells. A) HE, original magnification $10 \times$; B) $\mathrm{HE}$, original magnification $20 \times$; C) HE, original magnification $10 \times$. 
The following differential diagnosis was discussed:

- granulomatosis with polyangiitis,

- tuberculosis infection,

- cholesteatoma.

Laboratory testing revealed increasing levels of nonspecific markers of inflammation (erythrocytes sedimentation rate - ESR and C-reactive protein - CRP), serum creatinine level and urine test results were normal. The specified tests anti-neutrophil cytoplasmic antibodies on indirect immunofluorescence ANCA were negative, as well as anti-PR3 and anti-MPO autoantibodies. Mantoux test for tuberculosis was performed and it was negative, as well as a subsequent QuantiFERON test. Cultures and stains of the biopsy were negative for infectious organisms. An X-ray of the lungs and ultrasound examination of the kidneys were observed as well, with no abnormalities.

The patient has had a consultation with a rheumatologist because a diagnosis of possible ANCA-associated vasculitis, granulomatosis with polyangiitis (limited form) was suggested, based on the histological result (granulomatous necrotising inflammation). Despite the radical surgical therapy the symptoms of the patient (ear discharge and headache) persisted. The facial nerve palsy has slowly recovered.

The patient presented to the Clinic of Rheumatology. Therapy with methylprednisolone $60 \mathrm{mg} /$ day was started and azathioprine $100 \mathrm{mg} /$ day was added to his treatment regimen. After starting the therapy on a routine examination after two weeks the patient had no complaints from the ENT organs. The methylprednisolone dosage was tapered slowly, and azathioprine (100 mg/day) was continued. Five months after the beginning of the therapy the patient came with a second attack of granulomatosis with polyangiitis, again on the same mastoid process. A revision mastoidectomy was performed. The ESR and CRP values were elevated, and the anti CANCAs antibody titre was positive $1: 40$ (normal $1: 20$ ), and anti-PR3 antibodies $23 \mathrm{U} / \mathrm{ml}$ (normal $<5 \mathrm{U} / \mathrm{ml}$ ). Pulse therapy with methylprednisolone $(1000 \mathrm{mg})$ and cyclophosphamide (1000 mg) was started monthly. Methylprednisolone dosage was increased to $60 \mathrm{mg} /$ day and azathioprine was continued. One month later, the ESR and CRP normalised with concurrent improvement of the symptoms. The methylprednisolone dosage between the pulses was tapered slowly, and azathioprine was continued.

\section{Clinical case 2}

The second patient, a 44-year-old man, presented to the otolaryngologist with sudden purulent secretion from the right ear with $20 \mathrm{~dB}$ conductive hearing loss. After two weeks of unsuccessful antibiotic course, the patient presented with ipsilateral (right) facial nerve palsy and acute right-side headache. Computed tomography and magnetic resonance tomography of the head showed acute mastoiditis of the right mastoid process. After a "wall down" mastoidectomy and the following therapy with corticosteroids and mannitol, the patient recovered from the pain and the facial palsy. The histological result showed unspecific inflammation.

The following differential diagnosis was discussed:

- unspecific inflammation of the mastoid process,

- granulomatosis with polyangiitis,

- tuberculosis.

Laboratory testing revealed increasing levels of nonspecific markers of inflammation (erythrocytes sedimentation rate - ESR and C-reactive protein), serum creatinine level and urine test results were normal. The specified tests anti-neutrophil cytoplasmic antibodies on indirect immunofluorescence ANCA were positive 1 : 80 (normal < $1: 20$ ) as well as anti-PR3 autoantibodies $9.4 \mathrm{U} / \mathrm{ml}$ (normal $<5 \mathrm{U} / \mathrm{ml}$ ). Mantoux test for tuberculosis was performed and it was negative as well as a subsequent QuantiFERON test. The other examinations: X-ray of the lungs, echocardiography, and ultrasound of the kidneys did not show pathological features. A consultation was made with a rheumatologist, who concluded that the patient should be followed-up in the subsequent three months because the patient recovered after the operation and there were insufficient criteria for granulomatosis with polyangiitis.

Two months later the patient presented to the otolaryngologist with a perforation of the left tympanic membrane, granulations in the nose, granulations on the right conjunctiva, and formation in the epipharynx (Fig. 3A-D).

The otolaryngologist made a biopsy of the formation in the epipharynx because the histological result showed clusters of lymphocytes, macrophages, and fibroblast, forming granulomas without necrosis. The specified tests: anti-neutrophil cytoplasmic antibodies on indirect immunofluorescence anti ANCA antibodies, were positive $1: 160$ (normal $<1: 20$ ) as well as anti-PR3 autoantibodies under $100 \mathrm{U} / \mathrm{ml}$ (normal $<5 \mathrm{U} / \mathrm{ml}$ ). A diagnosis of granulomatosis with polyangiitis (limited form) was defined. The patient presented to the Clinic of Rheumatology with complaints of acute headache and pain in the left mastoid process. Magnetic resonance tomography of the head showed pansinusitis, otitis media chronica of the left ear, granulations in the epipharynx, and enlarged lymph nodes. Due to suspicion of superimposed infection, antibiotic therapy was started - vancomycin $2 \mathrm{~g} /$ day i.v., ciprofloxacin $2 \times 400 \mathrm{mg}$ i.v., and antifungal therapy with fluconazole 200 mg/day i.v. Methylprednisolone was started in a dose of $60 \mathrm{mg}$ daily. Pulse therapy 

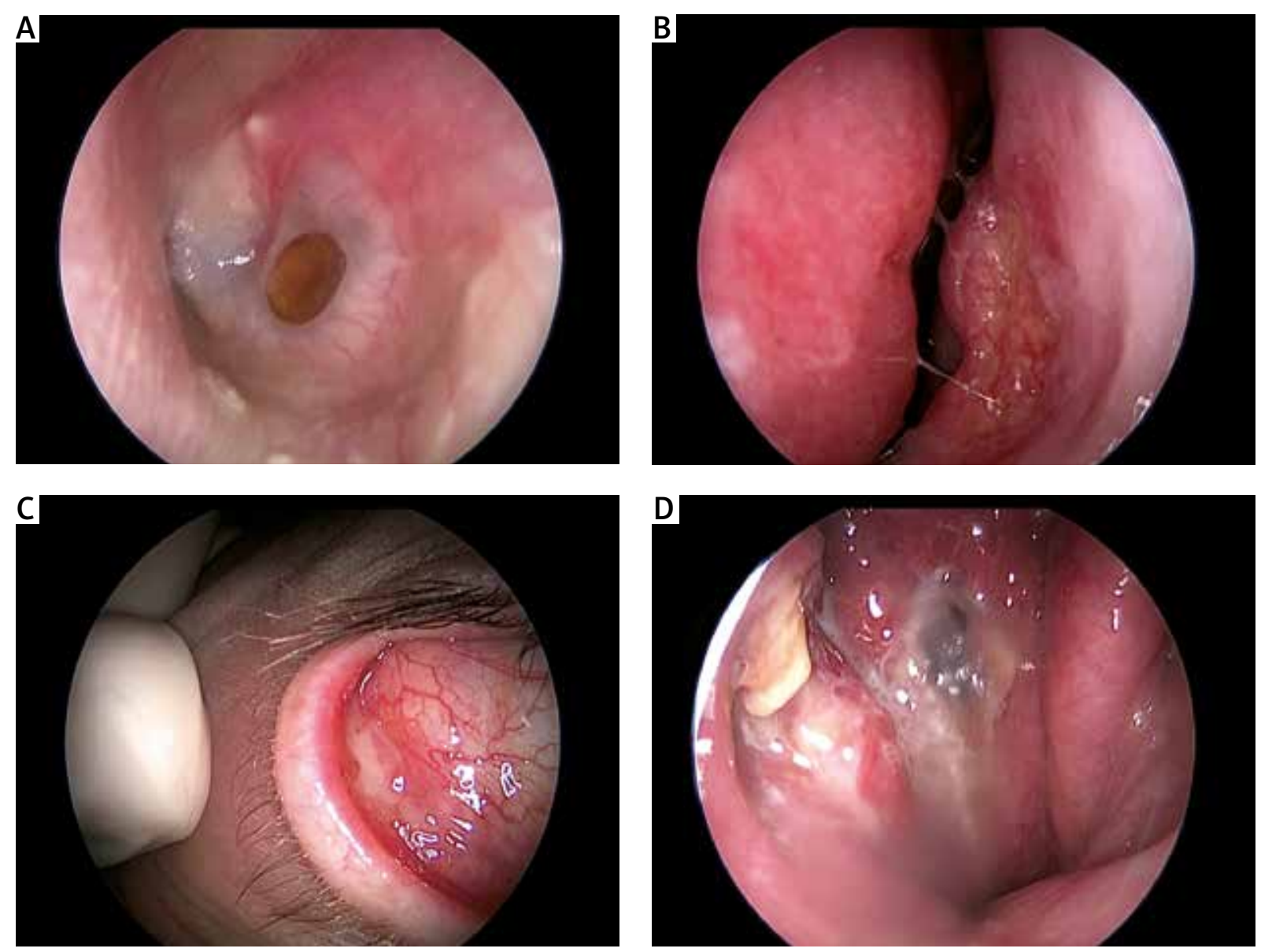

Fig. 3. Central perforation of the tympanic membrane of the left ear (A). Granulation in the left nasal cavity (in front of the inferior turbine) (B). Conjunctival granulation (C). Granulations in the epipharynx (D).

with intravenous immunoglobulin (400 mg/kg i.v.) was applied. After the end of the antibiotic course, the therapy with MabThera (rituximab) was made. One month later the patient's symptoms resolved and the immunological findings (CANCA, anti-PR3 autoantibodies) have been reduced.

\section{Discussion}

Granulomatosis with polyangiitis is a primary systemic small vessel vasculitis with predilection for the respiratory tract and kidney [1]. Two forms of granulomatosis with polyangiitis may be distinguished: systemic/ generalised forms and localised/limited forms [3]. These forms seem also to differ histologically, with systemic GPA being associated more often with vasculitis lesions and localised GPA with marked granulomatous features [4]. The limited/localised forms represent less than onethird of all GPA cases at diagnosis [4].

By far the most utilised classification system is the 1990 American College of Rheumatology (ACR) criteria for the classification of vasculitis. The ACR 1990 classification criteria for Wegener's granulomatosis include [5]:

- nasal or oral inflammation (painful or painless oral ulcers or purulent, bloody nasal discharge),

- abnormal chest radiograph showing nodules, fixed infiltrates, or cavities,

- abnormal urine sediment (microscopic haematuria with or without red cell casts),

- granulomatous inflammation on biopsy of an artery or perivascular area.

Two from four criteria are required for classification (sensitivity $88 \%$ and specificity $92 \%$ ). According to the definition of vasculitides, adopted by the Chapel Hill Consensus, Wegener's granulomatosis is characterised by granulomatous inflammation involving the respiratory tract, and necrotising vasculitis affecting small to medium-sized vessels (capillaries, veins, arterioles, and arteries) [8]. The typical histological features in granulomatosis with polyangiitis are presented by vasculitis, involving small vessels, granulomatous inflammation, and "geographic" necrosis because usually this histological 
picture is found in biopsies of the kidney and lung. In contrast to biopsies of the kidney and lung, tissue samples from involved areas of the upper respiratory tract (nose, sinuses, and subglottic region) are frequently non-diagnostic, yielding non-specific acute and chronic inflammation in up to $50 \%$ of biopsies. Upper respiratory tract biopsies demonstrate the full pathologic triad of granulomatous inflammation, vasculitis, and necrosis only in about $15 \%$ of cases [4].

The aim of the article was to present two clinical cases of limited form of granulomatosis with polyangiitis, and the most important purpose of this article is to focus on the isolated middle ear presentation of granulomatosis with polyangiitis. The common feature between the cases were similar clinical manifestations, presented by otitis media with acute mastoiditis and peripheral facial nerve palsy, because the pathologic process affected only middle ear cavity and mastoid and the histological results showed non-specific granulomatous inflammation without vasculitis.

The middle ear cavity and mastoid were obliterated by granulomatous tissue because the biopsy showed a marked granulomatous necrotising inflammation in the first clinical case. There were no traces of granulomatous inflammation within the wall of an artery or in the perivascular area (vasculitis) and affection of the upper respiratory tract. Non-specific inflammation of the middle ear cavity and mastoid was found in the second case at the beginning. The patient developed granulomatous inflammation of the upper respiratory tract (nose, epipharynx) two months later. According to the ACR 1990 classification criteria and Chapel Hill Consensus, there were insufficient criteria for the diagnosis granulomatosis with polyangiitis in two clinical cases at the beginning.

The middle ear is an anatomic structure, which is not part of the upper respiratory tract. But the embryonic development of the middle ear mucosa originates from the endoderm of the first pharyngeal pouch, or the middle ear and pharynx have an identical origin [9]. For the last 20 years, the middle ear mucosa has been recognised to be essentially a respiratory of modified respiratory mucosa [9]. That assertion gives reason to assign middle ear granulomatous inflammation to the inflammation of the upper respiratory tract. We suggest referral of granulomatous inflammation of the middle ear and mastoid to the classification criteria for granulomatosis with polyangiitis. On the other hand, the middle ear mu- cosa and mastoid have a small amount of blood vessels, which could explain the lack of vasculitis in the biopsy of the middle ear and mastoid. In conclusion, provided that strongly suggestive clinical disease manifestations are present, the finding of even only parts of the triad in an upper respiratory tract biopsy may argue compellingly for the diagnosis granulomatosis with polyangiitis.

Early diagnosis and treatment of patients with granulomatosis with polyangiitis define favourable prognosis. Involvement of the middle ear and mastoid carries risks of intracranial complications, so the right diagnosis is very important for early as well as late prognosis of the disease [2, 3]. In cases of otitis media with mastoiditis caused by granulomatosis with polyangiitis, the surgical treatment has limited success. Immunosuppressive therapy has the best effect in those patients. But the treatment of granulomatosis with polyangiitis (corticosteroids and immunosuppressive therapy) has various side effects, and so the "ex juvantibus" therapy is hazardous.

The authors declare no conflict of interest.

\section{References}

1. Stone JS. Wegener's granulomatosis. Rheumatology. Hochberg M, Silman A, Smolen J, et al. (eds.). Fifth ed, 2012; 1547-1557.

2. Costa CF, Polanski JF. Wegener's granulomatosis. Arch Otorhinolaryngologia 2015; 19; 266-268.

3. Trimarchi M, Sinico RA, Teggi R, et al. Otorhinolaryngological manifestations in granulomatosis with polyangiitis (Wegener's granulomatosis). Autoimmun Rev 2012; 12; 501-505.

4. Devaney K, Travis W, Hoffman G, et al. Interpretation of head and neck biopsies in Wegener's granulomatosis. A pathological study of 126 biopsies in 70 patients. Am J Surg 1990; 14: 555.

5. Hunder GG, Arend WP, Bloch DA, et al. The American College of Rheumatology 1990 Criteria for the classification of vasculitis. Introduction. Arthritis Rheum 1990; 33: 1065-1067.

6. Bloch DA, Michel BA, Hunder GG, et al. The American College of Rheumatology 1990 Criteria for the classification of vasculitis. Patients and methods. Arthritis Rheum 1990; 33: 1068-1073.

7. Fires JF, Hunder GG, Bloch DA, et al. The American College of Rheumatology 1990 Criteria for the classification of vasculitis. Summary. Arthritis Rhem 1990; 33: 1135-1136.

8. Jenette JC, Falk RJ, Andrassy K, et al. Nomenclature of systemic vasculitides: proposal of an International Consensus Conference. Arthritis Rheum 1994; 37: 187-192.

9. Smith ME. The Pharynx and upper airway. In: Rudolph's pediatrics. Rudolph CD, Rudolph AM, Lister GE, et al. (eds.). $22^{\text {nd }} \mathrm{ed}$. McGraw-Hill Companies, New York 2017. 